\title{
Factores Psicosociales Asociados a la Adherencia al Tratamiento de la Diabetes Mellitus Tipo 2.
}

\author{
Psychosocial Factors Associated with Adherence to Treatment of Type 2 Diabetes Mellitus
}

\author{
Manuel Ortiz \\ Eugenia Ortiz \\ Alejandro Gatica \\ $\mathrm{y}$ \\ Daniela Gómez \\ Universidad de La Frontera, Chile
}

(Rec: 28 de julio de 2009; Acep: 17 de septiembre de 2010)

\begin{abstract}
Resumen
Se han reportado bajas tasas de adherencia al tratamiento de enfermedades crónicas (50\%). Asimismo, los factores psicosociales estarían relacionados con la adherencia al tratamiento. Con el propósito de identificar la relación que existe entre factores psicosociales y la adherencia al tratamiento de la DM2 en usuarios del sistema de salud pública chileno, se diseñó un estudio no experimental transversal correlacional ( $\mathrm{n}=50$, edad promedio 60,76 años). Se autoadministraron encuestas para evaluar estrés y estilo de afrontamiento, sintomatología depresiva, percepción de apoyo social y adherencia al tratamiento. $40 \%$ de los pacientes presenta valores de hemoglobina glicosilada (Hb1 Ac) mayores a 9\%, mientras que un $66 \%$ de los participantes presenta desde moderados a altos niveles de estrés. En promedio durante los últimos 7 días los participantes han cumplido con la dieta general en 4,37 días, han consumido frutas y verduras en 3,14 días y han realizado exámenes de glicemia en 1,1 días. Existe una correlación directa entre el estrés y la hemoglobina glicosilada. El apoyo social y la sintomatología depresiva no se asociaron con la adherencia al tratamiento pero sí con el estrés. Se confirman pobres cifras de adherencia, y la asociación entre alto estrés y altos índices de Hb1Ac.

Palabras clave: Diabetes mellitus tipo 2, adherencia al tratamiento, estrés, sintomatología depresiva, estilos de afrontamiento
\end{abstract}

\begin{abstract}
It is estimated that rates of non-adherence to chronic diseases are around 50\%. International studies have reported some psychosocial factors related to treatment adherence. The purpose of the present study was to detect the relationship between psychosocial factors and treatment adherence in a sample of Type 2 Diabetics subjects from a public health institution. To this end, a non - experimental transversal correlational study was designed with a 50 DM2 subjects sample, which average age was 60,76 years old. Self-report measures of stress level, coping style, depressive symptoms, social support perception and treatment adherence, were obtained. The results revealed that $40 \%$ of participants had glycosylated hemoglobin over $9 \%$, and $66 \%$ of them shown moderate to high stress levels. In average during the last 7 days, the participants have accomplished the diet 4,37 days, consumed fruits and vegetables 3,14 days and took glycemia test in 1,1 days. There is a direct correlation between the stress level and concentrations of glycosylated hemoglobin. Unlike stress, social support and depressive symptomatology was not associated with treatment adherence. These results confirm low levels of treatment adherence and high rates of stress in DM2 patients, as well as its association with high levels of glycosylated hemoglobin.

Key words: Type 2 diabetes mellitus, treatment adherence, psychological stress, depression, copying style
\end{abstract}

Este estudio se enmarca en la etapa de pilotaje del proyecto del Fondo Nacional de Investigación y Desarrollo en Salud, FONIS N SA07I20071, que lleva por título "Factores psicosociales asociados a la adherencia al tratamiento de pacientes diabéticos tipo 2 de la ciudad de Temuco".

Correspondencia: Manuel Ortiz P. Fono: (45) 325552 Fax: (45) 341480. E-mail: msortiz@ufro.cl 


\section{Introducción}

El estudio de la adherencia a los tratamientos de las enfermedades crónicas resulta especialmente relevante, pues se estima que en la actualidad las cifras de la no adherencia bordean el 50\% (Martín \& Grau, 2005; Silva, Galeano \& Correa, 2005). En el caso de la DM2, la no adherencia al tratamiento tiene efectos a corto plazo (hiperglicemia) y largo plazo (complicaciones micro y macrovasculares tales como pie diabético, retinopatía y falla renal) (Gallegos, Cárdenas \& Salas, 1999; García \& García de los Ríos, 1992). Además, impide evaluar la efectividad de los tratamientos, impacta negativamente en la calidad de vida del paciente (Martín \& Grau, 2005), ocasiona al Estado altos costos económicos por pérdida de productividad, recursos asociados a rehabilitación y pensiones de invalidez.

Los principales ejes del tratamiento de la diabetes descritos incluyen educación diabetológica, recomendaciones nutricionales, ejercicio y autocontrol. La multidimensionalidad del mismo, el esfuerzo que implica modificar hábitos previamente establecidos así como la disposición para mantenerlos, permiten afirmar que el tratamiento es complejo, más aún si se considera que los adultos son más resistentes al cambio (Papalia, Wendkos \& Duskin, 2005).

Diversos estudios han demostrado que existen factores psicológicos y sociales relacionados con la adherencia al tratamiento de la DM (Chlebowy \& Garvin, 2006; Di Matteo, 2004; Ortiz, 2004; Ortiz, 2006; Paddison, Alpass, \& Stephens, 2008; Sacco \& Yanover, 2006), tales como estrés y sus estilos de afrontamiento, sintomatología depresiva y percepción de apoyo social.

En pacientes con DM, el estrés tiende a estar más presente desde los inicios de la enfermedad y a lo largo de todo su curso, ya que se considera estresante el solo hecho de padecer una enfermedad metabólica, crónica e incapacitante. El estrés está relacionado significativamente con un pobre control glicémico en pacientes diabéticos (Guthrie, Bartsocas, Jarros - Chabot \& Konstantinova, 2003; Peyrot, McMurray \& Kruger, 1999; Polonsky, Fisher, Earles \& Dudl, 2005). Lazarus \& Folkman (1986) establecen dos estilos básicos de afrontamiento: el centrado en la emoción (con objeto de cambiar el significado de una situación estresante y para regular el resultado negativo de las emociones) y el centrado en el problema (con objeto de cambiar o controlar algunos aspectos de una situación percibida como estresante). Al respecto, pareciera que las estrategias de afrontamiento al estrés que tenga un paciente se relacionaran con la adherencia al tratamiento. Es así como Peyrot, McMurray \& Kruger (1999) demostraron que ambos estilos de afrontamiento pueden estar asociados con el control glicémico. Ortiz (2006) sugiere que aquellos sujetos que afrontan conductualmente presentan un mejor control metabólico que aquellos que lo hacen emocionalmente. Por su parte Boland \& Grey (1996) sugieren que centrarse en el afecto se asocia con un pobre control metabólico, incluyendo el no cumplimiento del régimen terapéutico.

Con respecto a la sintomatología depresiva, se ha demostrado que puede afectar el control glicémico, la disminución de la actividad física, el aumento de obesidad, junto con potenciar las complicaciones asociadas al daño del organismo (Lin, Katon, von Korff, Rutter, Simon, \& Oliver, 2004). La prevalencia de la depresión en la población diabética es de un 23,6\%, comparado con un 17,1\% de la población no diabética (Goldney, Phillips, Fisher, \& Wilson, 2004).

El apoyo social es otra variable que estaría asociada con la adherencia a los tratamientos (Coffman, 2008; Di Matteo, 2004; van Dam et al., 2005), ya que entregaría al paciente mayores recursos para afrontar el evento estresante y de este modo conducir a un mejor control de las glicemias (Lo, 1999), aumentando la adherencia al tratamiento y reduciendo el estrés (Wiesli, Schmid, Kerwer, Nigg - Koch, Klaghofer, \& Seifert, 2005). Göz, Karaoz, Goz, Ekiz \& Cetin (2007) reportan una relación estadísticamente significativa entre apoyo social y calidad de vida de pacientes DM2.

Tomando en consideración los antecedentes teóricos y empíricos antes señalados, el propósito de este estudio fue evaluar los niveles de adherencia de pacientes diabéticos tipo 2 de la ciudad de Temuco e identificar la relación que existe entre la adherencia al tratamiento y las variables de apoyo social, estrés, estilos de afrontamiento y depresión.

\section{Método}

\section{Participantes}

Por medio de un muestreo no probabilístico, se seleccionaron 50 participantes con DM2, usuarios del Consultorio Miraflores de la ciudad de Temuco, bajo los siguientes criterios de inclusión: a) pacientes diagnosticados con DM2; b) usuarios del Consultorio Miraflores; c) firma de consentimiento informado.

Se recolectaron datos en la sala de espera del Consultorio Miraflores de la ciudad de Temuco. Para resguardar y cautelar el bienestar de los participantes, se cumplieron los principios éticos que dicta la American Psychological Association (1992).

\section{Instrumentos}

Apoyo social percibido: Medido a través de la Escala multidimensional de percepción de apoyo social (MSPSS) de Zimet, Dahlem, Zimet, \& Farley (1988), validada en la Región de La Araucanía por Ortiz \& Baeza (2011). Por medio de 12 ítems evalúa el apoyo social de otros significativos (pareja), la familia y el grupo de pares. Para los 12 ítems, el coeficiente de confiabilidad alfa de Cronbach fue 0,87. 
Sintomatología depresiva: Evaluada con la escala de depresión del centro de estudios epidemiológicos CES - D. Contiene 20 ítems con un formato de respuesta tipo Likert. El coeficiente de confiabilidad alfa de Cronbach para los 20 ítems fue 0,918, conservándose los ítems originales.

Estrés: Se utilizó la escala de estrés para diabéticos de Polonsky et al., (2005). Cuenta con 17 ítems con un formato de respuesta tipo Likert. El coeficiente de confiabilidad alfa de Cronbach para los 17 ítems fue 0,93.

Estilo de afrontamiento: Evaluado mediante la escala de Carver, Scheier \& Weintraub (1989). Este instrumento tiene un formato tipo Likert con respuestas que van desde 0 (En absoluto), hasta 3 (En gran medida). El coeficiente de confiabilidad alfa de Cronbach para los 27 ítems originales fue de 0,84 .

Adherencia al tratamiento: Fue evaluada directamente con el Summary of Diabetes Self-Care Activities de Toobert, Hampson \& Glasgow (2005). Este instrumento consta de 11 ítems que miden las conductas de autocuidado de los pacientes en los últimos 7 días. Los ítems dan lugar a 6 subcategorías: dieta general, dieta específica, ejercicio, automonitoreo, cuidado de pies y consumo de cigarrillos. El coeficiente de confiabilidad osciló entre 0,393 y 0,853, eliminándose el ítem 6 de la subcategoría de ejercicio y analizándose en forma individual los ítems de dieta específica, tal como lo sugieren los autores de la escala.

Además, la adherencia al tratamiento fue evaluada indirectamente con el último registro de hemoglobina glicosilada presente en las fichas médicas, al igual que los parámetros de presión arterial y peso.

\section{Análisis estadístico}

Frecuencias y datos descriptivos fueron obtenidos a través del Software SPSS 15.0. Para probar las hipótesis sobre la asociación de factores psicosociales y la adherencia al tratamiento de la DM2 se realizaron correlaciones de Pearson. Para comparar grupos se ejecutó la prueba ANOVA de un factor.

\section{Resultados}

\section{Descripción de la muestra}

El promedio de edad de los participantes fue 60,76 años (DT $=9,57) .38 \%$ corresponde al género masculino. El promedio de la hemoglobina glicosilada fue de 9,110 (DT $=2,83$ ), encontrándose que un $26 \%$ de la muestra presentó un buen control metabólico (valores de hemoglobina glicosilada bajo 7\%), un 34\% presentó un control moderado (hemoglobina glicosilada entre 7 y $8,9 \%$ ) y el restante $40 \%$ tuvo un pobre control metabólico (valores iguales o mayores a 9\%). La media del peso de los sujetos fue de 78,26 kilos (DT = 13,29), de la presión sistólica fue de 137,16 (DT = $34,21)$, de la presión diastólica fue de 77,80 (DT = 9,35) y de la última glicemia registrada en la ficha del paciente fue de 213,76 (DT = 83).

Se obtuvieron tres categorías de estrés: bajo estrés, estrés moderado y elevado estrés, cuyas frecuencias fueron de $34 \%, 36 \%$ y $30 \%$ respectivamente (ver tabla 1 ).

Tabla 1:

\begin{tabular}{lcrrrr}
\multicolumn{7}{c}{ Descripción de los participantes } \\
\hline & $\mathrm{N}$ & Mínimo & Máximo & Media & Desv. típ. \\
\hline Edad (años) & 50 & 32 & 78 & 60,76 & 9,578 \\
Hemoglobina glicosilada (\%) & 50 & 4,9 & 16,7 & 9,110 & 2,8339 \\
Peso (Kilogramos) & 50 & 52,0 & 107,0 & 78,262 & 13,2941 \\
Presión sistólica (mmHg) & 50 & 100 & 240 & 137,16 & 34,219 \\
Presión diastólica (mmHg) & 50 & 60 & 112 & 77,80 & 9,357 \\
Glicemia (mg/dl) & 50 & 99 & 397 & 213,76 & 83,004 \\
N válido (según lista) & 50 & & & & \\
\hline
\end{tabular}

\section{Adherencia al tratamiento}

En la subcategoría de dieta general, el promedio de días de autocuidado en la última semana fue de 4,37 (DT $=2,31$ ), en la subcategoría de dieta específica, el ítem referente al consumo de frutas y verduras tuvo una media de $3,14(\mathrm{DT}=3,024)$ y el ítem concerniente al consumo de alimentos con grasa tuvo una media de 5,56 (DT $=1,960)$. En la subcategoría de ejercicio, el promedio fue de 4,80 (DT $=2,650)$; el promedio de automonitoreo en los últimos siete días fue de $1,10(\mathrm{DT}=1,64)$ y en la subcategoría de cuidado de pies la media fue de 4,56 (DT = 2,39). Sólo 8 de los participantes reportaron haber fumado en la última semana un promedio de 8,5 cigarrillos (DT $=7,63$ ).

Estos resultados fueron contrastados por medio de una prueba ANOVA de un factor, utilizando como variable de clasificación los tres grupos conformados de acuerdo a su control metabólico (bueno, moderado y pobre control metabólico), no encontrando diferencias estadísticamente significativas en ninguna de las conductas pilares que requiere el tratamiento (ver tabla 2). 
Tabla 2:

Promedios de conductas de los últimos siete días de la semana, según la escala adherencia "Summary of Diabetes Self-Care Activities".

\begin{tabular}{|c|c|c|c|c|c|}
\hline & Buen control & Moderado control & Pobre control & Media muestra total & $p$ \\
\hline Dieta general & 4,8 & 4,4 & 4,02 & 4,3700 & 0,639 \\
\hline Automonitoreo glicemia & 3,61 & 4,76 & 4,47 & 1,1000 & 0,415 \\
\hline Cuidado de pies & 4,96 & 4,58 & 4,27 & 4,5600 & 0,731 \\
\hline Consumo frutas y Verduras & 2,54 & 4,06 & 2,75 & 3,14 & 0,305 \\
\hline Consumo de alimentos con grasa & 4,69 & 5,47 & 6,2 & 5,56 & 0,132 \\
\hline $\begin{array}{l}\text { Ejercicio al menos } 30 \text { minutos } \\
\text { últimos } 7 \text { días }\end{array}$ & 5,54 & 4,18 & 4,85 & 4,80 & 0,383 \\
\hline
\end{tabular}

Valores expresados en días durante la última semana

\section{Correlación de variables}

Como se observa en la tabla 3 , los sujetos que presentaron un mayor nivel de estrés presentaron bajos niveles de adherencia al tratamiento, específicamente en la subcategoría de dieta general, en donde la correlación fue significativa y negativa $(r=-0,354 ; p=0,012)$, a su vez, estrés se relacionó directamente con la variable hemoglobina glicosilada $(r=$ 0,$291 ; p=0,040$ ).

No se encontraron relaciones estadísticamente significativas entre la sintomatología depresiva, apoyo social percibido y estilos de afrontamiento con la adherencia al tratamiento sin embargo, se encuentran en la dirección esperada según lo postulado teóricamente.

En cuanto a las correlaciones encontradas, se puede mencionar que a mayor nivel de hemoglobina glicosilada mayor es el consumo de alimentos con grasa $(r=0,292 ; p=$ $0,039)$. La variable estrés se vincula de forma negativa con la variable apoyo social percibido $(r=-0,526 ; p=0,000)$ $\mathrm{y}$ de forma positiva con la sintomatología depresiva $(r=$
$0,517 ; p=0,000)$. Por su parte, mientras mayor es el apoyo social percibido, menor es la sintomatología depresiva $(r=$ $-0,648 ; p=0,000$ ).

Dentro de las conductas de autocuidado, aquellos sujetos que presentan mayor cumplimiento de la dieta general tienen mayores frecuencias de conductas de automonitoreo de glicemia $(r=0,330 ; p=0,019)$; en la subcategoría de dieta específica, a mayor consumo de frutas y verduras, menor es el consumo de alimentos con grasa $(r=-0,403 ; p=0,004)$ y el consumo de frutas y verduras se asocia positivamente a la variable apoyo social percibido $(r=0,290 ; p=0,041)$.

En cuanto a los estilos de afrontamiento, aquellos sujetos con mayor tendencia al afrontamiento evitativo presentaron mayores niveles de estrés $(r=0,320 ; p=0,023)$ y mayor sintomatología depresiva $(r=0,302 ; p=0,033)$. Del mismo modo, aquellos sujetos que tienden mayoritariamente al afrontamiento centrado en la emoción presentaron mayor sintomatología depresiva $(r=0,613 ; p=0,000)$ y menor percepción de apoyo social $(r=-0,321 ; p=0,023)$ (ver tabla 3). 
Tabla 3:

Correlación entre variables del estudio.

\begin{tabular}{|c|c|c|c|c|c|c|c|c|c|c|c|c|}
\hline & $\begin{array}{l}\text { Hemoglobina } \\
\text { glicosilada } \\
\text { (1) }\end{array}$ & $\begin{array}{c}\text { Dieta } \\
\text { General. } \\
(2)\end{array}$ & $\begin{array}{l}\text { Automo- } \\
\text { nitoreo } \\
\text { (3) }\end{array}$ & $\begin{array}{l}\text { Frutas y } \\
\text { Verduras } \\
\text { (4) }\end{array}$ & $\begin{array}{c}\text { Comida } \\
\text { Grasa } \\
(5)\end{array}$ & $\begin{array}{c}\text { Estrés } \\
(6)\end{array}$ & $\begin{array}{c}\text { Depresión } \\
\text { (7) }\end{array}$ & $\begin{array}{c}\text { Apoyo } \\
\text { Social } \\
(8)\end{array}$ & $\begin{array}{l}\text { Afrontam. } \\
\text { Conductual } \\
\text { (9) }\end{array}$ & $\begin{array}{l}\text { Afrontam. } \\
\text { Cognitivo } \\
\text { (10) }\end{array}$ & $\begin{array}{l}\text { Afrontam. } \\
\text { Emocional } \\
\text { (11) }\end{array}$ & $\begin{array}{c}\text { Afrontam. } \\
\text { Evitativo } \\
\text { (12) }\end{array}$ \\
\hline $\begin{array}{l}\text { Hemoglobina } \\
\text { glicosilada } \\
\text { (1) }\end{array}$ & & $-0,203$ & 0,009 & $-0,046$ & $0,292 *$ & $0,291 *$ & 0,149 & $-0,190$ & $-0,077$ & 0,006 & $-0,114$ & $-0,067$ \\
\hline $\begin{array}{l}\text { Dieta } \\
\text { General. } \\
(2)\end{array}$ & & & $0,330 *$ & 0,097 & 0,216 & $-0,354 * *$ & $=0,018$ & 0,145 & $-0,077$ & 0,109 & 0,222 & 0,044 \\
\hline $\begin{array}{l}\text { Automo- } \\
\text { nitoreo } \\
\text { (3) }\end{array}$ & & & & 0,153 & 0,220 & $-0,188$ & 0,084 & 0,085 & $-0,014$ & $-0,084$ & 0,131 & 0,025 \\
\hline $\begin{array}{l}\text { Frutas y } \\
\text { Verduras } \\
\text { (4) }\end{array}$ & & & & & & 0,025 & $-0,032$ & $0,290^{*}$ & $-0,068$ & 0,005 & $-0,045$ & 0,142 \\
\hline $\begin{array}{l}\text { Comida } \\
\text { Grasa } \\
(5)\end{array}$ & & & & & & $-0,039$ & 0,200 & $-0,093$ & 0,179 & 0,045 & 0,235 & 0,065 \\
\hline $\begin{array}{l}\text { Estrés } \\
\text { (6) }\end{array}$ & & & & & & & $0,517 * *$ & $-0,526 * *$ & $-0,260$ & 0,097 & 0,183 & $0,320^{*}$ \\
\hline $\begin{array}{l}\text { Depresión } \\
\text { (7) }\end{array}$ & & & & & & & & $-0,648 * *$ & 0,029 & 0,210 & $0,613 * *$ & $0,302 *$ \\
\hline $\begin{array}{l}\text { Apoyo } \\
\text { Social } \\
\text { (8) } \\
\text { Afrontam. }\end{array}$ & & & & & & & & & 0,077 & $-0,205$ & $-0,321^{*}$ & $-0,144$ \\
\hline $\begin{array}{l}\text { Conductual } \\
\text { (9) }\end{array}$ & & & & & & & & & & $0,545^{* *}$ & $0,437 * *$ & 0,060 \\
\hline $\begin{array}{l}\text { Afrontam. } \\
\text { Cognitivo } \\
\text { (10) }\end{array}$ & & & & & & & & & & & $0,499 * *$ & 0,228 \\
\hline $\begin{array}{l}\text { Afrontam. } \\
\text { Emocional } \\
\text { (11) } \\
\text { Afrontam. } \\
\text { Evitativo } \\
(12) \\
\end{array}$ & & & & & & & & & & & & $0,419 * *$ \\
\hline
\end{tabular}

\section{Discusión}

Este estudio permitió constatar los pobres resultados del control metabólico que presentan los participantes. Se encontró que de 50 participantes, sólo $26 \%$ tenían un buen control metabólico y el restante $74 \%$ se distribuyen en un moderado (34\%) y pobre control metabólico $(40 \%)$. Este resultado podría explicarse examinando el comportamiento de los participantes con respecto a las conductas pilares del tratamiento. Ellos señalan adherir a la dieta recomendada por los especialistas en un promedio de 4,37 días a la semana, sin embargo, el consumo de alimentos que contienen grasas alcanza un promedio a la semana de 5,56 días, superando esta cifra a la dieta general recomendada, lo que además se relaciona con el bajo consumo de alimentos como frutas y verduras que alcanza una media de 3,14 días a la semana. Por otra parte, los factores específicos evaluados tales como conductas de autocuidado, práctica de actividad física, automonitoreo de glicemia y cuidado de los pies no se relacionaron significativamente con los índices de hemoglobina de los pacientes. 
Específicamente, el automonitoreo de glicemia es realizado en promedio sólo 1,1 días por semana. De tal comportamiento, se podría desprender que la mayoría de los participantes de la muestra no pueda evaluar la efectividad de su tratamiento, ni menos realizar ajustes en su tratamiento con miras a corregir posibles desequilibrios en sus indicadores metabólicos.

En cuanto a la actividad física, si bien el promedio de días en que los sujetos reportan practicar al menos media hora de ejercicio (incluyendo caminar) es de 4,80 días por semana, este valor no se asocia significativamente al índice de hemoglobina glicosilada, lo que podría explicarse porque los pacientes tienden a rotular como ejercicio conductas cotidianas que requieren un esfuerzo físico mínimo, tales como caminar en la casa o en el trabajo y por tanto, no involucrarse en conductas que promuevan la actividad física como uno de los ejes centrales del tratamiento de la DM2.

Con respecto al estrés, en este estudio se encontró que existe una correlación inversa de éste con el cumplimiento de la dieta general. Asimismo, altos niveles de estrés se asocian a altos niveles de hemoglobina glicosilada. Este hallazgo debe ser considerado a la luz de que se ha encontrado que un $66 \%$ de los participantes se situó entre la categoría de moderado y alto estrés, resultado esperable en el caso de pacientes DM2, debido al carácter crónico de la enfermedad y la complejidad del tratamiento (Paddison et al., 2008), lo que indica que este grupo de pacientes constituye un grupo de riesgo. Más aún si se considera que aquellos pacientes con altos niveles de estrés se centraron en el estilo de afrontamiento evitativo, lo cual de acuerdo a Lazarus \& Folkman (1986), no es la mejor estrategia para superar una situación estresante, generando a su vez mayores niveles de estrés.

Si bien las variables apoyo social percibido y sintomatología depresiva no están asociadas significativamente a la adherencia al tratamiento (con excepción de la correlación encontrada entre apoyo social y consumo de frutas y verduras), estas variables sí se vinculan de forma significativa con el estrés y entre sí, pudiendo asociarse indirectamente con los niveles de hemoglobina glicosilada, por medio del efecto que ejercen sobre el estrés. Este resultado merece mayor investigación y abre la puerta a futuras líneas de investigación que permitan una mejor comprensión de factores que puedan modular el efecto que tiene el estrés sobre marcadores fisiológicos y conductas de adherencia al tratamiento, posibilitando generar diferentes intervenciones sobre variables que inciden directamente en las conductas de autocuidado como el estrés, o sobre aquellas que pueden mediar los niveles de estrés de los pacientes diabéticos, como lo son el apoyo social percibido y la sintomatología depresiva.

Este estudio cuenta con limitaciones. Una de ellas guarda relación con el proceso de recolección de datos. Debido a las características de los participantes y el espacio físico en donde se llevó a cabo el proceso de recogida de datos, los encuestadores debieron recolectar la información en forma individual aumentando en forma considerable el tiempo destinado para tal proceso.

\section{Referencias}

American Psychological Association. (1992). Ethical principles of psychologists and code of conduct. American Psychologist, 47, 1597 - 1611.

Boland, E., \& Grey, M. (1996). Coping strategies of school - age children with diabetes mellitus. Diabetes Educator, 22, 592 - 597.

Carver, C., Scheier, M., \& Weintraub, J. (1989). Assessing coping strategies: A theoretically based approach. Journal of Personality and Social Psychology, 53, 267 - 283.

Chlebowy, D. O., \& Garvin, B. J. (2006). Social support, self-efficacy, and outcome expectations: Impact on self-care behaviors and glycemic control in Caucasian and African American adults with type 2 diabetes. Diabetes Educator, 32, 777-786.

Coffman, M. J. (2008). Effects of tangible social support and depression on diabetes self-efficacy. Journal of Gerontological Nursing, 34, 32-39.

Di Matteo, M. R. (2004). Social support and patient adherence to medical treatment: A meta - analysis. Health Psychology, 23, 207 - 218.

Gallegos, E., Cárdenas, V., \& Salas, M. (1999). Capacidades de autocuidado del adulto con diabetes tipo 2. Investigación y Educación en Enfermería, 17, 23 - 33.

García, H., \& García de los Ríos, M. (1992). Complicaciones crónicas de la diabetes. En M. García de los Ríos, G. López \& P. Durruty (Eds.), Diabetes Mellitus. Santiago: Arancibia Hnos

Goldney, R., Phillips, P., Fisher, L., \& Wilson, D. (2004). Diabetes, depression, and quality of life. Diabetes Care, 27, 1066 - 1070.

Göz, F., Karaoz, S., Goz, M., Ekiz, S., \& Cetin, I. (2007). Effects of the diabetic patient's perceived social support on their quality-of-life. Journal Of Clinical Nursing, 16, 1353 - 1360.

Guthrie, D., Bartsocas, C., Jarros - Chabot, P., \& Konstantinova, M. (2003). Psychosocial issues for children and adolescents with diabetes: Overview and recommendations. Diabetes Spectrum, 16, 7 - 12.

Lazarus, R., \& Folkman, S. (1986). Estrés y procesos cognitivos. Barcelona: Martínez Roca.

Lin, E., Katon, W. J., von Korff, M., Rutter, C., Simon, G., \& Oliver, M. (2004). Relationship of depression and diabetes self - care, medication adherence, and preventive care. Diabetes Care, 27, 2154 - 2160.

Lo, R. (1999). Correlates of expected success at adherence to health regimen of people with IDDM. Journal of Advanced Nursing, 39, $418-424$

Martín, L., \& Grau, J. (2005). La adherencia terapéutica como un problema de la psicología de la salud. En J. Grau (Ed.), Psicología de la Salud. Guadalajara: Universidad de Guadalajara.

Ortiz, M. (2004). Factores psicológicos y sociales asociados a la adherencia al tratamiento en adolescentes diabéticos tipo 1. Psykhe, 13, 21 - 31.

Ortiz, M. (2006). Estrés, estilo de afrontamiento y adherencia al tratamiento de adolescentes diabéticos tipo 1. Terapia Psicológica, 24, 139 - 147.

Ortiz, M., \& Baeza, M. (2011). Propiedades psicométricas de una escala para medir apoyo social percibido en pacientes diabéticos tipo 2 chilenos. Universitas Psychologica, 10, 65 - 72.

Paddison, C. A., Alpass, F. M., \& Stephens, C. V. (2008). Psychological factors account for variation in metabolic control and perceived quality of life among people with type 2 diabetes in New Zealand. International Journal of Behavioral Medicine, 15, 180-186.

Papalia, D., Wendkos, S., \& Duskin, R. (2005). Psicología del Desarrollo. México: Mc Graw Hill Interamericana.

Peyrot, M., McMurray, J., \& Kruger, D. (1999). A biopsychological model of glycemic control in diabetes: Stress, coping and regimen adherence. Journal of Health and Social Behavior, 40, 141 - 158.

Polonsky, W., Fisher, L., Earles, J., \& Dudl, R. (2005). Assesing psychological distress in diabetes: Development of the diabetes distress scale. Diabetes Care, 28, 626 - 638. 
Sacco, W. P., \& Yanover, T. (2006). Diabetes and depression: The role of social support and medical symptoms. Journal of Behavioral Medicine, 29, 523-531.

Silva, G., Galeano, E., \& Correa, J. (2005). Adherencia al tratamiento. Acta Médica Colombiana, 30, 268 - 273.

Toobert, D. J., Hampson, S. E., \& Glasgow, R. E. (2005). The summary of diabetes self - care activities measure. Diabetes Care, 23, 943 - 950.

van Dam, H. A., van der Horst, F. G., Knoops, L., Ryckman, R., Crebolder, H., \& van den Borne, B. (2005). Social support in diabetes: A syste- matic review of controlled intervention studies. Patient Education and Counseling, 59, 1 - 12.

Wiesli, P., Schmid, C., Kerwer, O., Nigg - Koch, C., Klaghofer, R., \& Seifert, B. (2005). Acute psychological stress affects glucose concentrations in patients with type 1 diabetes following food intake but not in the fasting state. Diabetes Care, 28, 1910 - 1915.

Zimet, G., Dahlem, N., Zimet, S., \& Farley, G. (1988). The multidimensional Scale of Perceived Social Support. Journal of Personality Assessment, 52, 30 - 41 